\title{
DIFFERENT APPROACHES IN SIMULATION PROPELLER OPEN WATER CHARACTERISTICS USING CFD METHOD
}

\author{
VU NGOC BICH \\ Ho Chi Minh City University of Transport, Vietnam
}

\begin{abstract}
The paper presents different approaches in simulation open water propeller performance by using CFD method, which are sliding mesh, moving reference frame and stationary reference frame approaches. The different in numerical setup, order of accuracy in simulation results and computational time between three methods are given. To confirm the accuracy of numerical results a well-known propeller of Japan Bulk Carrier ship model is selected for verification and validation process. At the end, the suggestion for choosing each approach based on the order of accuracy and computational time are given. During the research process, a commercial package provided by SIEMENS, Star-CCM+ was applied as a solver.
\end{abstract}

KEYWORDS: CFD, RANSE, Open Water \& Propeller

Received: Jun 09, 2020; Accepted: Jun 29, 2020; Published: Jul 27, 2020; Paper Id.: IJMPERDJUN2020482

\section{INTRODUCTION}

Recently, with the significant development of computational resources, many hydrodynamics problems in general can be solved by applying Computational Fluid Dynamics (CFD) method from the point of view level of accurate result and computational time compared to experimental approach [1-3]. In the application of CFD method for simulation open water propeller characteristics, there are many different approaches, i.e. Lifting Line Theories, Blade Element-Momentum Theory, Surface Panel Methods, Lifting Line Theories, Reynolds Averaged NavierStokes Equation (RANSE) [4]. For open-water propeller calculation, RANSE method is more popular approach compared with other approaches as its flow model is basically similar to actual flow physics. Therefore, in comparison with other potential flow approaches, RANSE provides more accuracy in numerical results [5], [6], [7].

Simulation open-water propeller characteristics using RANSE method is not an unpopular topic for researchers. Authors in [8-11], provided many useful results in the propeller open water performance prediction using RANSE method. Tu T.N. [11] studied the impact of different grid type (hexahedral grid and polyhedral grid) and turbulence models (SST K- $\omega$ and realizable $\mathrm{k}-\varepsilon$ two layer) on achieved numerical results of open-water propeller characteristics, and illustrated that applying hexahedral grid together with SST k- $\omega$ turbulence model may provide higher accuracy results. The thrust and torque coefficients errors vary from $0.8 \%$ up to $2 \%$ and $1.0 \%$ up to $2.5 \%$, respectively, for large advance coefficients J. Baltaza et al. [12] investigated the influence of discretization, and iterative errors, boundary conditions and computational domain size and numerical results in the case of open water. The results indicate that all of these factors effect on numerical results with different levels. Nakisa et al. [13] studied the impact of different turbulence models on numerical results of propeller open water 
characteristics and showed that the turbulence model SST K- $\omega$, combination with a sliding mesh provide the better results. The mean errors of thrust and torque coefficients are $7.5 \%, 9.7 \%$, respectively, compared to experimental results.

Those studies play significant role for researchers applying RANSE CFD approach to calculate propeller performance in open water condition. Nevertheless, those have not considered the impact of different methods on level accuracy of obtained numerical results as well as computational time. Therefore, these issues will be taken into account this paper. To confirm the accuracy of numerical results a well-known propeller of Japan Bulk Carrier ship model [14] is selected for verification and validation process. The simulation is performed with support commercial solver Star-CCM+.

The paper is organized as follows: Section 2 gives governing equations of three methods. Section 3 carried out numerical setup. Section 4 presents numerical obtained results with three different approaches. Finally, in Section 5 conclusions drawn from this research are discussed.

\section{MATERIALS AND METHODS}

As mentioned above, this study is performed with three approaches applying for open water propeller simulation: sliding mesh, moving reference frame and whole fluid domain rotation.

\section{Sliding Mesh Approach}

Sliding mesh (SM) approach is the method to describe the rotational motion of fluids. The Sliding mesh often uses when the time-accurate solution is desired. In the Sliding mesh technique, two computational domains are used. Those two computational domains are connected together: stationary domain and rotating domain. A domain is restrained by at least one interface zone where it meets the opposing cell domain. A grid interface is formed by the association between the interface zones of adjacent cell zones and other one [15]. The rotating domain rotates every time steps and the two domains' interface or connection is also re-computed one another time steps simultaneously. The main point in the Sliding mesh is about calculation of flux in and out the interface and searching for the matching cells after one time step (because the rotating domain has been rotated after one time step).

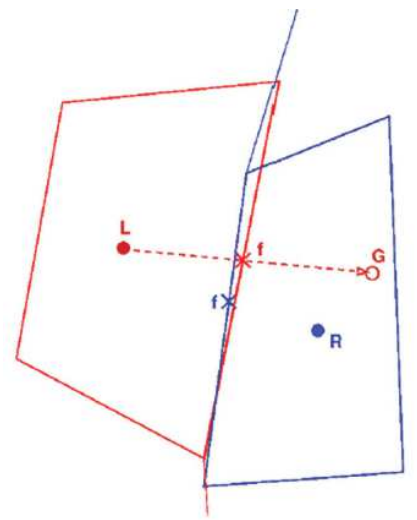

a)

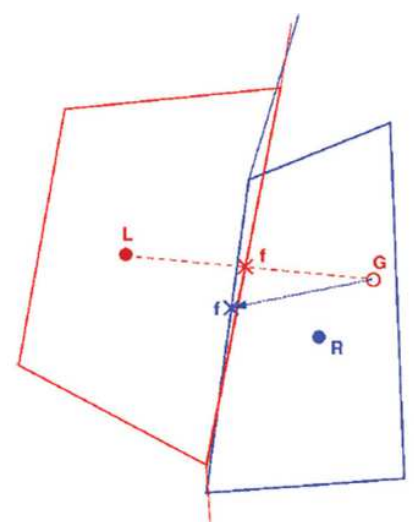

b)

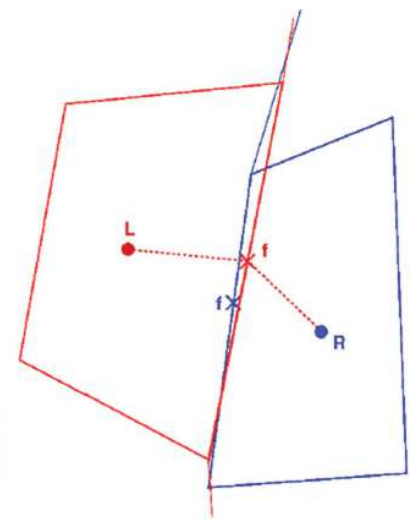

c)

Figure 1: Sliding faces: a -Construction of ghost points, b-searching the global faces, c-new neighbor cells [16]

The governing equation for sliding mesh is given as follows [15]:

$$
\frac{d}{d t} \int_{V} \rho \phi d V+\int_{\partial V} \rho \phi\left(\vec{u}-\vec{u}_{g}\right) d \vec{A}=\int_{\partial V} \Gamma \nabla \phi d\left(\vec{u}-\vec{u}_{g}\right) \vec{A}+\int_{V} S_{\phi} d V
$$


Where: $\rho$ is the fluid density; $\mathrm{V}$ is control volume; $\vec{u}$ is the velocity vector of flow; $\vec{u}_{g}$ is the mesh velocity of the moving mesh; $\phi$ is general scalar on an arbitrary control volume $\mathrm{V} ; \Gamma$ is the coefficient of diffusion; $S_{\phi}$ is the source term of $\phi ; \partial \mathrm{V}$ represents the boundary of the control volume $\mathrm{V} ; \vec{A}$ is face area vector.

\section{Moving Reference Frame Method}

Moving reference frame (MRF) approach provides a manner of modeling rigid translations and rotations as a steady-state problem, and leaving the grid stationary at the same time. Rotating reference frames can rigidly move, or rotate and translate, corresponding to the laboratory frame. In [16], the velocity of fluid particles in moving frame, ${ }^{r_{r}}$ is defined as following:

$$
\vec{v}_{r}=\vec{v}-\vec{u}_{r}
$$

Where: $\vec{u}_{r}=\vec{\omega} \times \vec{r}_{r}$, is the velocity of fluid in rotating frame; $\vec{\omega}$ is rotating frame angular velocity; $\vec{v}$ is absolute velocity (observed in stationary frame).

By replacing $\vec{v}$ by $\vec{v}_{r}$ in rotating reference frame, the motion governing equation can be rewritten as follows:

$$
\begin{aligned}
& \nabla \times \rho \vec{v}_{r}=0 \\
& \nabla \times\left(\rho \vec{u}_{r} \vec{v}_{r}\right)+\rho\left(2 \vec{\omega} \times \vec{v}_{r}+\vec{\omega} \times \vec{\omega} \times \vec{r}\right)=-\nabla \rho+\mu \nabla \times \nabla\left(\vec{v}_{r}\right)
\end{aligned}
$$

As can be seen in momentum equation (4), the additional terms $\left(2 \vec{\omega} \times \vec{v}_{r}\right)$ and $\vec{\omega} \times \vec{\omega} \times \vec{r}$ represent Carioles term and centripetal acceleration term respectively. Observing from the moving reference frame the propeller will stand still.

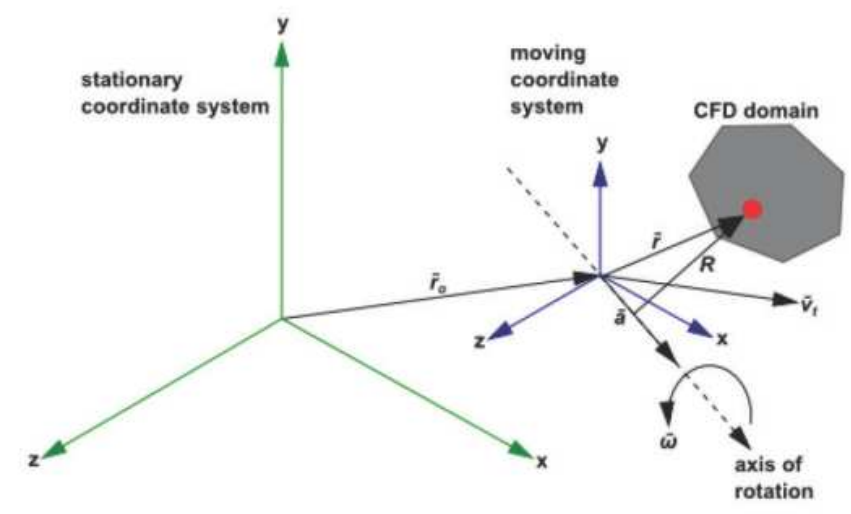

Figure 2: Description of moving reference frame approach

\section{Stationary Reference Frame Approach}

Stationary reference frame (SRF) approach is the classical way to simulate open water propeller like in actual towing tank, which simulated real propeller revolution in the domain. It means that there is only one domain. Propeller rotates in the computational domain. The governing equation is still Navier Stokes equation.

\section{Comparison of Three Approaches in the Physical Point of View}

From the view of MRF, the flow around propeller can be considered as a steady-state problem versus MRF. Therefore, 
during the simulation, the solver does not need to do the iterations for each time step. It is also possible to use unsteady simulation with moving reference frame method and whole fluid domain rotation for open water simulation, to capture some unsteady behavior of flow during the simulation, such as vortex shedding. However, in this paper, the rotating reference frame method has been performed with unsteady simulation to get more accuracy of obtained result, compared with steady simulation. Sliding grid and Stationary reference frame approaches are unsteady simulation, so that for each time step, iterations need to be perform to reach convergence state. Moreover, with Sliding grid method, after each time step, the solver also has to do: search algorithm and interpolation for sliding nodes; as well as flux computation at the sliding interface. Thus, the Rotating reference frame is expected to be less time consumption, and the Sliding mesh takes the most computational time.

\section{NUMERICAL SETUP}

\section{Reference propeller model}

The propeller model under study in this paper is a propeller of Japan Bulk Carrier ship model. All the necessary data of flow physics and CFD validation of this has been provided in [14]. The principal particulars and geometry of propeller are presented in Figure.3 and Table 1.

Table 1: Propeller geometry of JBC

\begin{tabular}{|l|c|c|c|}
\hline \multicolumn{2}{|c|}{ Description } & Unit & Value \\
\hline \multicolumn{1}{|c|}{ Propeller diameter } & D & m & $\mathbf{0 . 2 0 3}$ \\
\hline Blade area ration & AE/A0 & - & 0.50 \\
\hline Pitch ration & P0.7/D & - & 0.75 \\
\hline Hub ration & Dh/D & - & 0.18 \\
\hline Number of blades & Z & - & 5 \\
\hline Propeller rotating direction & - & - & Right-handed \\
\hline
\end{tabular}

\section{Computational Domain and Boundary Conditions}

One of the main factors affecting the obtained numerical results is the size of computational domain and boundary conditions. In open-water propeller simulation, the computation domain size should be determined to ensure the uniform the propeller incoming flow upstream of and to eliminate any propeller reflections downstream. The size of computational domain around the propeller was set with respect to the recommendations of International Towing Tank Conference (ITTC) [17].

For Moving reference frame and Stationary reference frame approaches, the size of computational domain are defined as follow: The inlet and outlet boundaries were located at a distance of 4D and 6D from propeller plane, respectively. The outlet boundary was located at a distance $8 \mathrm{D}$ from the propeller axis (see Figure 4). 


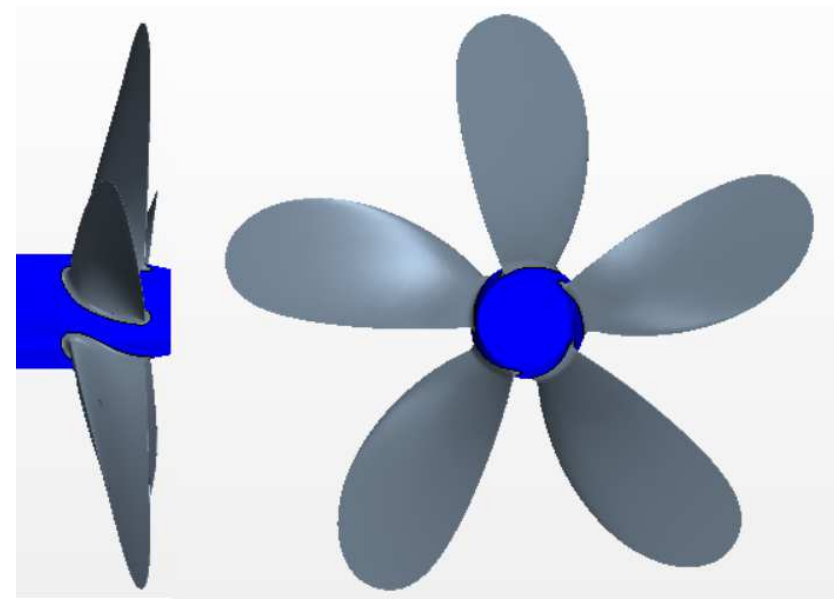

Figure 3: The propeller geometry of Japan Bulk Carrier ship model

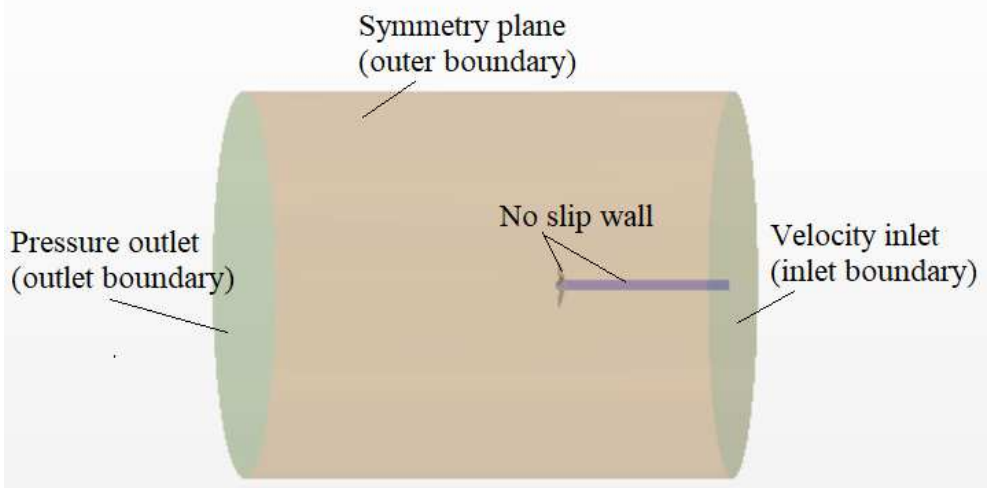

Figure 4: Calculation domain and boundary conditions for MRF and SRF methods

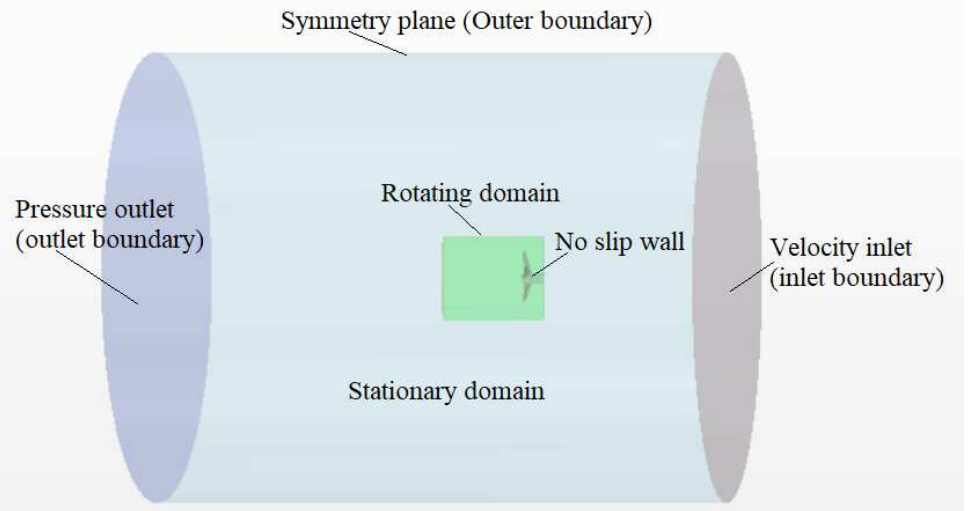

Figure 5: Calculation domain and boundary conditions for SM method

In Sliding mesh approach, the simulation domains are divided in two types: one is the rotating domain inside the stationary domain (Figure 5), the other is outer domain which has same dimension as MRF and SRF methods. However, the dimension of inner one should cover at least the whole propeller inside. There are common faces between these two domains, which is called "None matching connection face." It is not required that, the grid of this face is matched point-to point with each other. Using that connection face, the solver can be able to compute flux through two domains. For each time step, the position of inside domain changes corresponding to its rotation. Therefore, this connection should be recalculated each time step.

- The boundary conditions is set for all three methods as follows: 
- Inlet boundary: velocity inlet condition with advance velocity imposed;

- Outlet boundary: Pressure outlet condition;

- Outer boundary: Symmetry plane condition;

- Solid parts (propeller blade, hub and shaft): No-slip wall condition.

\section{Mesh Generation}

The mesh type used for calculations is hexahedral cells. It is applied for MRF, SRF and Sliding mesh methods. The mesh is generated by a specifying base mesh size corresponding to all spacing defined. It was wasteful to apply fine mesh in unnecessary location (far from the propeller), therefore the local volume refinements was created around the propeller itself. Moreover, the regions of tip of propeller blades, trailing and leading edges are much more refined as blade surface has large curvature in these regions [8]. To observe the exact flow behavior near the propeller walls, prism layer was applied. The total cells number used for MRF and SRF methods are around 2.8 million cells and for sliding mesh method is 3.2 million cells. The result of mesh generation for three methods are shown in Figure.6 and 7.
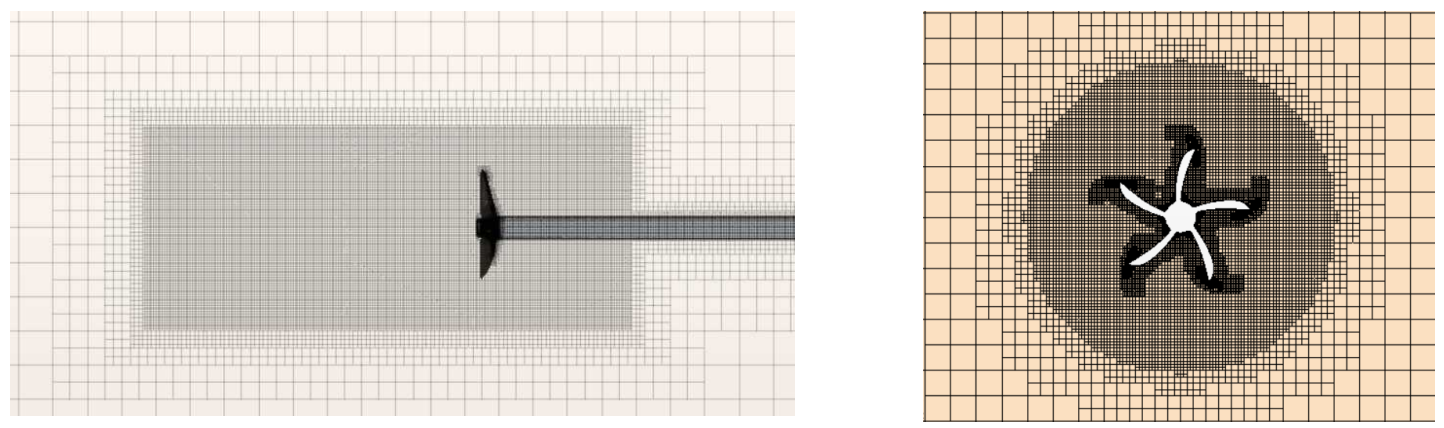

Figure 6: Result of mesh generation for MRF and SRF methods
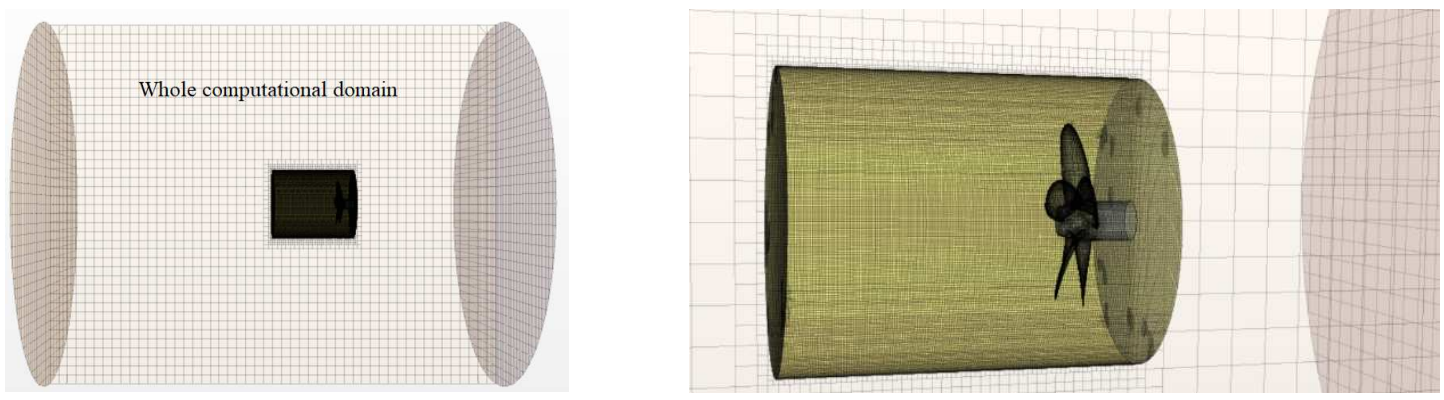

Figure 7: Result of mesh generation for Sliding mesh method

\section{Physics Model}

Viscous unsteady RANSE was applied in computation for all three methods (Sliding mesh, MRF and methods). The turbulence model applied for all three methods in the computations was SST K- $\omega$ two equation-model as it is capable of resolving two additional equations for the eddy viscosity, which consist of turbulence kinetic energy (k) and dissipation rate (typically $\varepsilon$ or $\omega$ ). This model has proven to be capable to predict ship hydrodynamics accurately. Another reason is that these are the most popularly applied by CFD researchers (according to statistics of the submissions for the Gothenburg 2010 Workshop, $80 \%$ of these used k- $\omega$ two equation-model) [18]. 
In setup process of these methods, the major differences between each one are the time step and the number of iteration per time step. This input setup may directly impacts on time consumption or computer resources during simulation. The MRF method can be recognized as a steady approach for open-water test, therefore it is recommended to use large time step and small numbers of iteration. For sliding mesh method and SRF method the time step was chosen so that the rotation angle of propeller equals to one degree per time step [19]. Detail setup of time step in Table 2. Computation of the numerical simulation is carried out parallel on cluster with 24 cores.

Table 2: Time step setup for open water propeller simulation

\begin{tabular}{|l|c|c|}
\hline \multicolumn{1}{|c|}{ Method } & Number of Iteration per time step & Time step \\
\hline Sliding mesh & 5 & $1.5 .10-4 \mathrm{~s}$ \\
\hline MRF & 10 & $1.10-2 \mathrm{~s}$ \\
\hline SRF & 5 & $1.5 .10-4 \mathrm{~s}$ \\
\hline
\end{tabular}

\section{RESULTS AND DISCUSSIONS}

When convergence is reached, the result is obtained by estimating the force in $\mathrm{X}$ direction (propeller thrust) and the moment about $\mathrm{X}$ axis (propeller torque) on propeller blades and hub. The thrust and torque are described in nondimensional forms by Thrust coefficient $\left(\mathrm{K}_{\mathrm{T}}\right)$ and Propeller torque coefficient $\left(\mathrm{K}_{\mathrm{Q}}\right)$. Then, the efficiency of open water $\left(\eta_{0}\right)$ is defined as follows:

$$
\begin{aligned}
& K_{T}=\frac{T}{\rho n^{2} D^{4}} \\
& K_{Q}=\frac{Q}{\rho n^{2} D^{5}} \\
& J=\frac{V_{A}}{n D} \\
& \eta_{0}=\frac{J}{2 \pi} \frac{K_{T}}{K_{Q}}
\end{aligned}
$$

\begin{tabular}{|c|c|c|c|c|c|c|c|c|c|}
\hline \multirow{2}{*}{$\mathbf{J}$} & \multicolumn{3}{|c|}{ KT } & \multicolumn{3}{|c|}{$10 K Q$} & \multicolumn{3}{|c|}{$\eta 0$} \\
\hline & CFD & EFD [20] & E\%D & CFD & EFD [20] & E\%D & CFD & EFD [20] & E\%D \\
\hline \multicolumn{10}{|c|}{ Sliding mesh method } \\
\hline 0.4 & 0.2257 & 0.2214 & -1.94 & 0.2867 & 0.2871 & 0.14 & 0.5012 & 0.5143 & 2.55 \\
\hline 0.5 & 0.1812 & 0.1798 & -0.78 & 0.2452 & 0.2479 & 1.09 & 0.5881 & 0.5868 & -0.22 \\
\hline 0.6 & 0.1369 & 0.1349 & -1.48 & 0.2009 & 0.2027 & 0.89 & 0.6507 & 0.6388 & -1.87 \\
\hline 0.7 & 0.09 & 0.0867 & -3.81 & 0.1478 & 0.1509 & 2.05 & 0.6784 & 0.6547 & -3.62 \\
\hline 0.8 & 0.039 & 0.0371 & -5.12 & 0.0872 & 0.0921 & 5.32 & 0.5695 & 0.522 & -9.09 \\
\hline \multicolumn{10}{|c|}{ b) MRF method } \\
\hline 0.4 & 0.2248 & 0.2214 & -1.54 & 0.2882 & 0.2871 & -0.38 & 0.4966 & 0.5143 & 3.45 \\
\hline
\end{tabular}

Where: $\mathrm{T}-$ thrust of propeller $[\mathrm{N}]$; Q-torque of propeller $[\mathrm{Nm}] ; \mathrm{V}_{\mathrm{A}}$ - advance speed $\left[\mathrm{m} \cdot \mathrm{s}^{-1}\right]$; $\mathrm{n}-$ propeller revolution $[\mathrm{rps}] ; \rho$ - fluid density $\left[\mathrm{kg} \cdot \mathrm{m}^{-3}\right]$.

Table 3: Open water propeller simulation results of different approach compared to experimental data 


\begin{tabular}{|l|c|c|c|c|c|c|c|c|c|c|}
\hline 0.5 & 0.1768 & 0.1798 & 1.67 & 0.2462 & 0.2479 & 0.69 & 0.5715 & 0.5868 & 2.61 \\
\hline 0.6 & 0.138 & 0.1349 & -2.30 & 0.2002 & 0.2027 & 1.23 & 0.6582 & 0.6388 & -3.04 \\
\hline 0.7 & 0.0908 & 0.0867 & -4.73 & 0.153 & 0.1509 & -1.39 & 0.6612 & 0.6547 & -0.99 \\
\hline 0.8 & 0.0355 & 0.0371 & 4.31 & 0.0938 & 0.0921 & -1.85 & 0.4819 & 0.522 & 7.69 \\
\hline \multicolumn{10}{|c|}{ c) SRF method } \\
\hline 0.4 & 0.2188 & 0.2214 & 1.17 & 0.2877 & 0.2871 & -0.21 & 0.4842 & 0.5143 & 5.86 \\
\hline 0.5 & 0.1789 & 0.1798 & 0.50 & 0.2502 & 0.2479 & -0.93 & 0.5690 & 0.5868 & 3.03 \\
\hline 0.6 & 0.1317 & 0.1349 & 2.37 & 0.2032 & 0.2027 & -0.25 & 0.6189 & 0.6388 & 3.11 \\
\hline 0.7 & 0.0855 & 0.0867 & 1.38 & 0.154 & 0.1509 & -2.05 & 0.6185 & 0.6547 & 5.52 \\
\hline 0.8 & 0.0391 & 0.0371 & -5.39 & 0.0872 & 0.0921 & 5.32 & 0.5709 & 0.522 & -9.37 \\
\hline
\end{tabular}

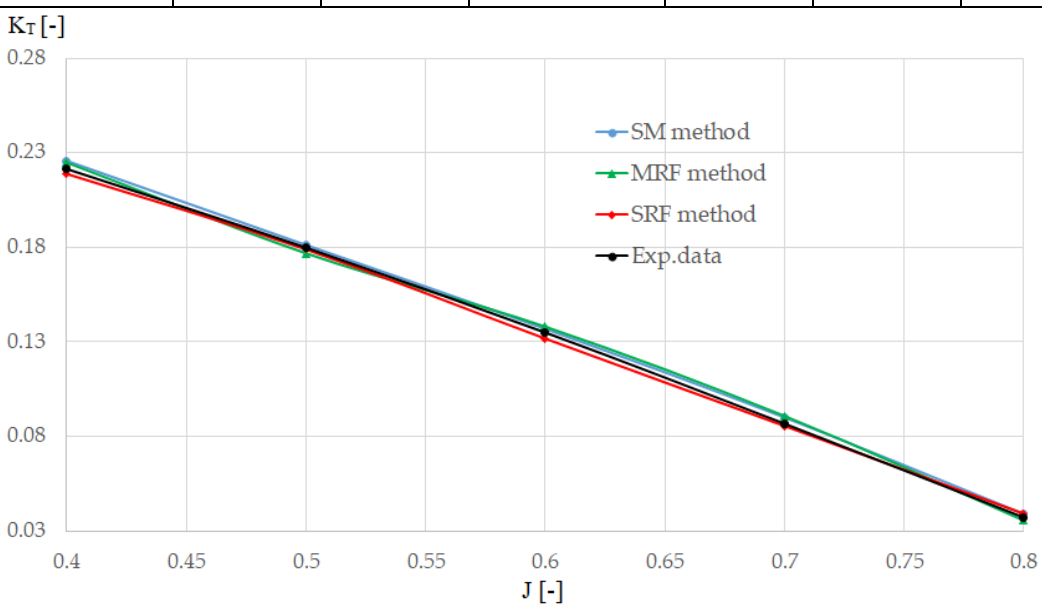

Figure 8: Thrust coefficients obtained from 3 different methods, comparing with experiment data (EFD)

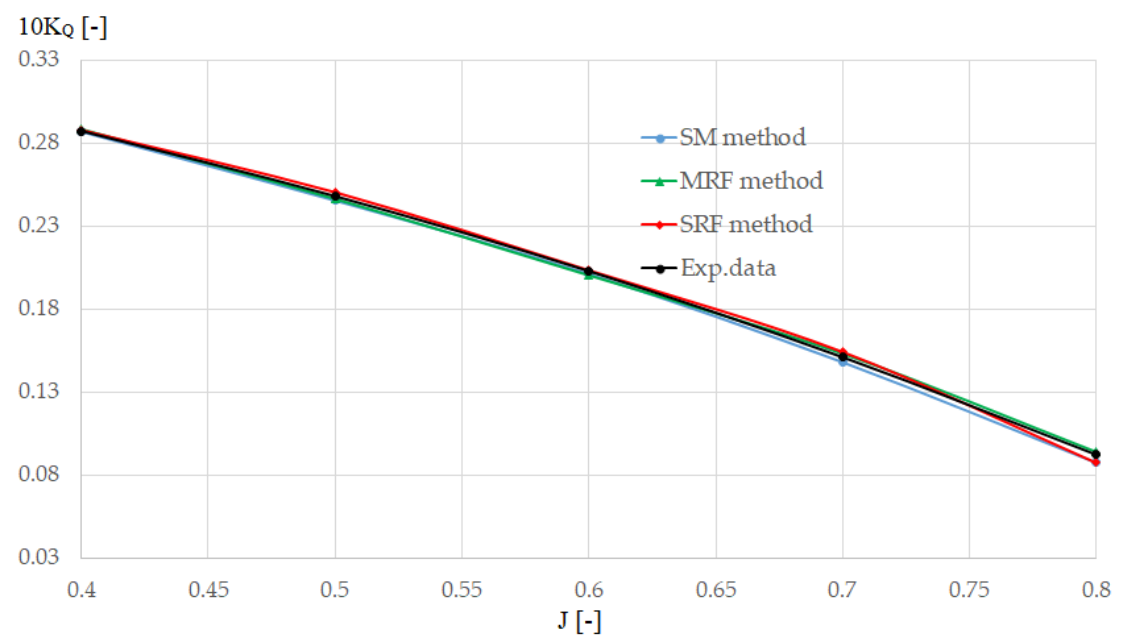

Figure 9: Torque coefficients obtained from 3 different methods, comparing with experiment data (EFD) 


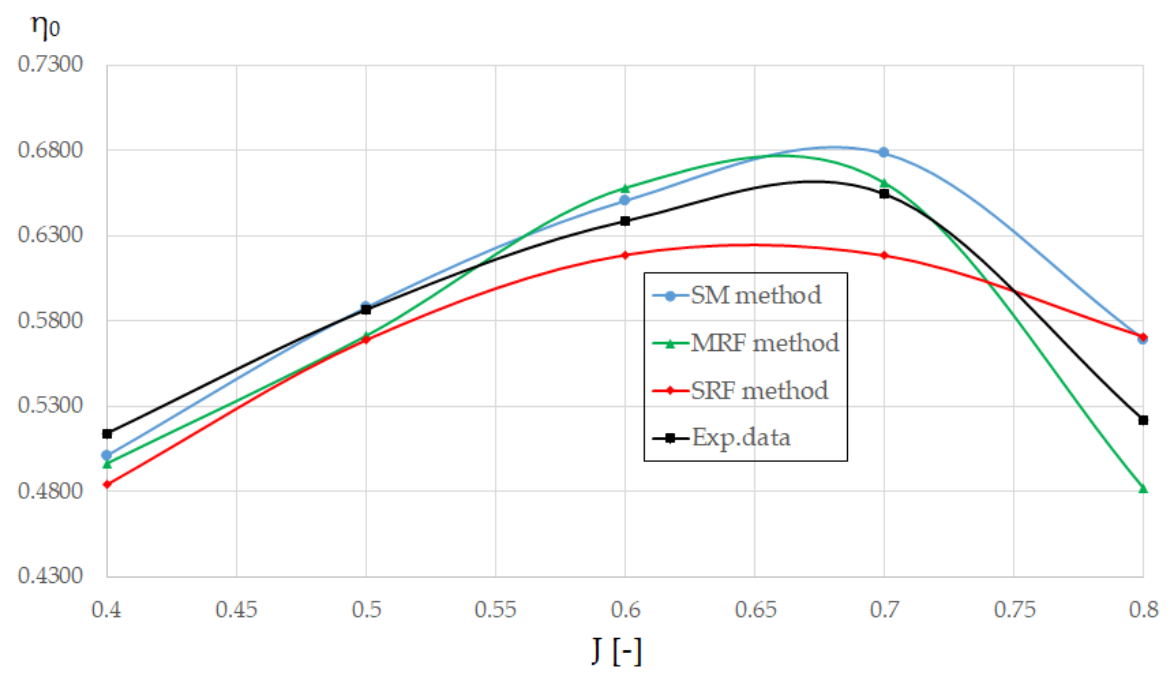

Figure 10: Efficiency of open water obtained from 3 different methods, comparing with experiment data (EFD)

Table 3 and Figure. 8, 9, 10 and 11 present the comparison between predicted and experimented characteristics of propeller open water using different approaches. The discrepancy between CFD, S and the EFD (Experimental Fluid Dynamic) data, D is defined as follow:

$$
E \% D=\frac{(D-S)}{D} \cdot 100 \%
$$
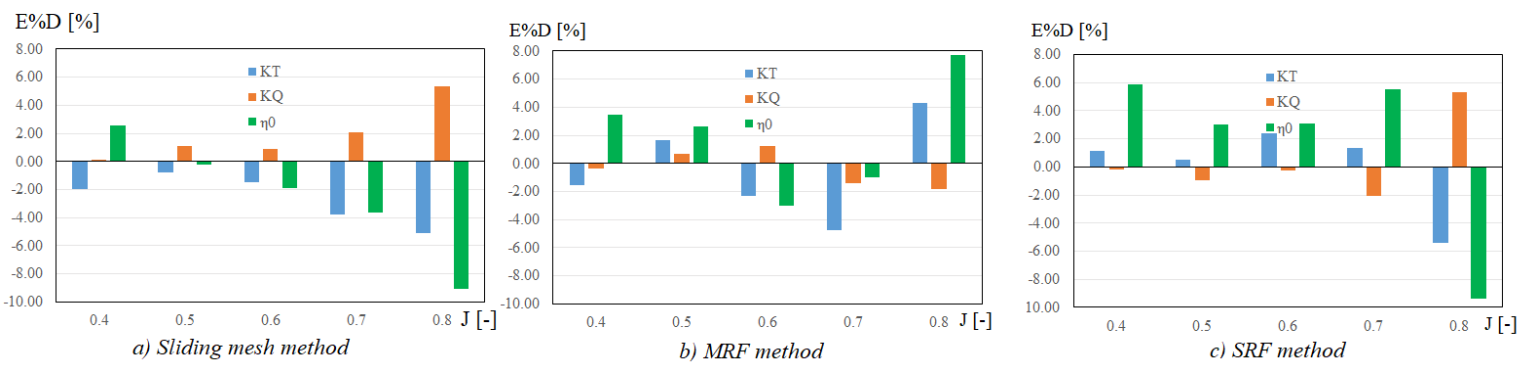

Figure 11: Open water propeller simulation results of different approach in comparison with experimental data

Generally, in the aspect of numerical result among three methods, the numerical obtained results show that, there is only small differences between these. The simulation results of all approaches are good at $\mathrm{J}$ from 0.4 to 0.7 particularly, from $0.5 \%$ to $5.5 \%$ difference for all $\mathrm{K}_{\mathrm{T}}, \mathrm{K}_{\mathrm{Q}}$, and $\eta_{\mathrm{O}}$. The higher difference occurred when $\mathrm{J}$ is ranged from 0.8 up to $5 \%$ and $9 \%$ for $\mathrm{K}_{\mathrm{T}}, \mathrm{K}_{\mathrm{Q}}$ and $\mathrm{J}$, respectively. The differences in obtained results using three different methods can be explained by different in Total pressure coefficient distribution on blades at suction and pressure sides for different simulation approaches (see Fig.12 and Fig.13). 


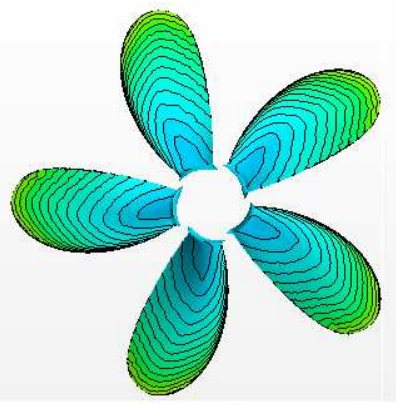

a) Sliding mesh method

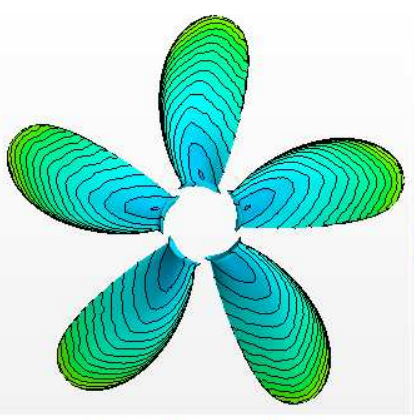

b) MRF method

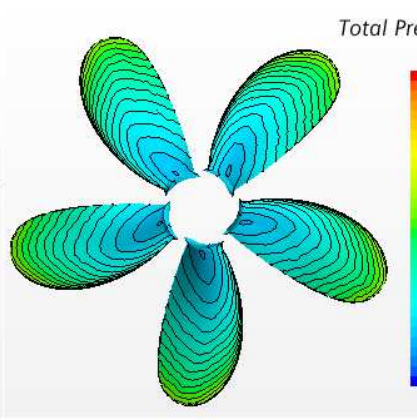

c) SRF method

Figure 12: Total pressure coefficient distribution at $\mathbf{J}=\mathbf{0 . 7 0}$ on blades of propeller at suction side for different simulation approaches

Table 4 shows the results of calculation time per time step for different numerical approaches. It is clear that Moving reference frame takes least computational time, by less than one-third in comparison with sliding mesh method. Therefore, moving reference frame method is the helpful method in open water propeller simulation from the point of view order of accuracy as well as computational time.

The diffident in computational time between three methods can be explained as follow:

First, the computation run time significantly depends on the setup of time steps and the convergence of the simulation. The values of time step present how rapid the propeller rotates during the computation process. The number of iteration inside each time step also plays a vital role to the computational time and the level of accuracy of obtained result. However, the problem of finding suitable setting for two values above can only be solved by practical use, or trial and error. The authors have start with roughly time step of 1 degree per time step and test the result with different setup of time steps. With two methods, Sliding mesh and SRF, in case of increasing time steps, and decreasing the number of iteration per time step, the results get worse, leading to the under prediction of the forces. Beside, larger time step makes the force have higher oscillation. The influence of time steps to open water propeller simulation has been studied by Zhan-zhi Wang et al. [21].

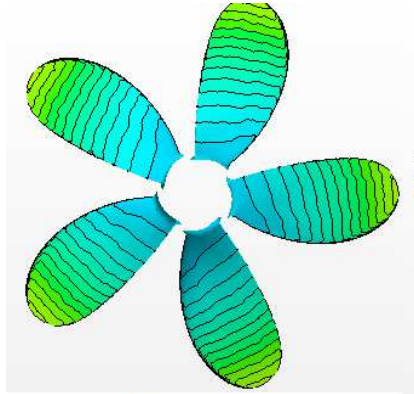

a) Sliding mesh method

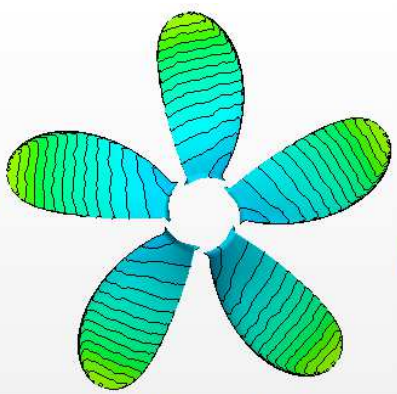

b) MRF method

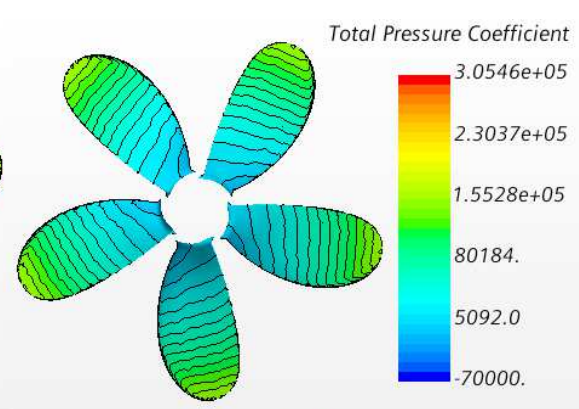

c) SRF method

Figure 13: Total pressure coefficient distribution at $\mathbf{J}=\mathbf{0 . 7 0}$ on blades of propeller at pressure side for different simulation approaches

Table 4: Computational time of different approaches

\begin{tabular}{|l|c|c|}
\hline Different approaches & Time elapsed per time step (average) (s) & $\begin{array}{c}\text { Percentage (compared to } \\
\text { sling mesh method) }\end{array}$ \\
\hline Sling mesh method & $10.0 \mathrm{~s}$ & $100 \%$ \\
\hline MRF method & $6.7 \mathrm{~s}$ & $67.0 \%$ \\
\hline SRF method & $2.8 \mathrm{~s}$ & $28 \%$ \\
\hline
\end{tabular}


Secondly, in the theoretical points of view, prediction of propeller forces and moments in open water case is a transient problem. In SM method, the solver has to calculate the flux through the Sliding interface for each time steps, when the SRF does not. Therefore, the computational time of Sliding mesh is the largest one. Besides, as stated above, even if unsteady simulation is used for the solver with MRF method, this method still has a steady-state formulation. So, the setup of time step with MRF method makes it run with less computational time and still maintaining the good level of accuracy in comparison with SRF. The fluctuation of forces and moments with MRF method is also much smaller than two other methods.

\section{CONCLUSIONS}

In this paper, three approaches using RANSE solver are presented in simulation propeller open water performance. The following conclusions can be drawn from the result of the study:

- The good agreement between RANSE CFD and experimental data shows the capability of RANSE CFD in solving ship hydrodynamics problems in general and open water propeller performance in particular.

- There is only small difference in term of numerical result between three methods. However, there is significant difference in term of computational time between three methods.

- Moving reference frame method is the helpful method for prediction open water propeller characteristics from the point of view order of accuracy and computational time. Nevertheless, it is recommended that this method is compatible for one domain simulation, not for simulation of propeller behind the ship. In this case, Sliding mesh approach should be used.

\section{ACKNOWLEDGEMENTS}

The author is grateful to the Ho Chi Minh City University of Transport, Vietnam for providing necessary research facilities during conducting this research work.

\section{REFERENCES}

1. Drikakis, D., M. Frank, and G.J.E. Tabor, Multiscale computational fluid dynamics. 2019. 12(17): p. 3272.

2. Obeid, S., R. Jha, and G.J.F. Ahmadi, RANS simulations of aerodynamic performance of NACA 0015 flapped airfoil. 2017. 2(1): p. 2 .

3. Khan, Sher Afghan, Abdul Aabid, and Maughal Ahmed Ali Baig. "CFD analysis of CD nozzle and effect of nozzle pressure ratio on pressure and velocity for suddenly expanded flows." International Journal of Mechanical and Production Engineering Research and Development 8 (2018): 1147-1158.

4. Li, J., et al., Method for the Calculation of the Underwater Effective Wake Field for Propeller Optimization. 2019. 11(1): p. 165.

5. Molland, A.F., S.R. Turnock, and D.A. Hudson, Ship resistance and propulsion. 2017: Cambridge university press.

6. Brizzolara, S., D. Villa, and S. Gaggero. A systematic comparison between RANS and panel methods for propeller analysis. in Proc. Of 8th International Conference on Hydrodynamics, Nantes, France. 2008.

7. Paturu, Pallavi, et al. "Numerical Analysis of Flow Over NACA0012 at Fixed Mach Number Using Computational Fluid Dynamics." International Journal of Mechanical and Production Engineering Research and Development 7.6 (2017): 213222. 
8. Bertram, V., Practical ship hydrodynamics. 2011: Elsevier.

9. Perali, P., T. Lloyd, and G. Vaz. Comparison of URANS and BEM-BEM for propeller pressure pulse prediction: E779A propeller in a cavitation tunnel. in Proceedings of the 19th Numerical Towing Tank Symposium. 2016.

10. Felicjancik, J., et al., Numerical simulations of hydrodynamic open-water characteristics of a ship propeller. 2016. 23(4): $p$. $16-22$.

11. Gandhi Mallela, Pallavi Paturu, and M. Komaleswarao. "Lift and drag performance of NACA0012 airfoil at various angle of attack using CFD." International journals of mechanical and production engineering research and development 8.3 (2018): $89-100$.

12. Da-Qing, L.J.J.o.H., Ser. B, Validation of RANS predictions of open water performance of a highly skewed propeller with experiments. 2006. 18(3): p. 520-528.

13. Baltazar, J.M., D.R. Rijpkema, and J.F. De Campos. Numerical studies for verification and validation of open-water propeller RANS computations. in Proceedings of the 6th International Conference on Computational Methods in Marine Engineering (Rome, Italy). 2015.

14. Rasheed, Wakkas, Sahib Ahmed, and Nabeel Ghyadh. "Investigation Potential Flow About Curved Wing Using Panel Method." International Journal of Mechanical and Production Engineering Research and Development (IJMPERD) ISSN (P) (2016): 2249-6890.

15. Tu, T.N.J.A.E.J., Numerical simulation of propeller open water characteristics using RANSE method. 2019. 58(2): p. $531-537$.

16. Baltazar, J.M., D.R. Rijpkema, and J. Falcao De Campos. Numerical studies for verification and validation of open-water propeller RANS computations. in Proceedings of the 6th International Conference on Computational Methods in Marine Engineering (Rome, Italy). 2015.

17. Nakisa, M., M.J. Abbasi, and A.M. Amini. Assessment of marine propeller hydrodnamic performance in open water via CFD. in Proceedings of The 7th International Conference on Marine Technology (MARTEC 2010). 2010.

18. https://t2015.nmri.go.jp/Instructions_JBC/instruction_JBC.html.

19. Ansys Fluent Theory Guide, Ansys Inc 2018.

20. Numeca Fine Marine, Theory Manual, 2013.

21. ITTC-Recommended Procedures and Guidelines. Practical Guidelines for Ship Self-Propulsion CFD. 2014.

22. Yong, Z., et al., Turbulence model investigations on the boundary layer flow with adverse pressure gradients. 2015. 14(2): $p$. 170-174.

23. ITTC 2014. Practical Guidelines for Ship Self-Propulsion CFD Available from: https://www.ittc.info/media/8169/75-03-03o1.pdf.

24. https://t2015.nmri.go.jp/Instructions_JBC/Case_1-5a.html.

25. Wang, Z.-z. and Y.J.C.o.e. Xiong, Effect of time step size and turbulence model on the open water hydrodynamic performance prediction of contra-rotating propellers. 2013. 27(2): p. 193-204. 